\title{
Preface to Special Issue on Photocatalysis in China
}

Semiconductor photocatalytic reactions are, by definition, light-induced reactions driven by surface photogenerated electron-hole pairs. Photogenerated electrons, with strong reduction ability, can be utilized for hydrogen production from splitting water, as well as solar-fuel emergency from $\mathrm{CO}_{2}$ reduction. Meanwhile, photogenerated holes, with powerful oxidation ability, also become judicious choice for disinfection, pollutant contamination and self-cleaning coating. Photocatalytic technology becomes prevalent solution for environmental purification and solar-energy conversion. They have found unprecedented opportunities and garnered great interest in environmental, energy, building materials and chemical industries. The investigation on them in China is at the frontline in comparison to its international counterparts. The quantity of "Essential Science Indicators (ESI) highly-cited papers" over the past decade was 2169. Among which, mainland Chinese researchers have published 1185 papers in total; while American and Japan have published 428 and 151 papers, respectively. Obviously, Chinese occupies the highest share (54.6\%), followed by American (19.7\%) and Japan (7\%). Apart from the quantity, the citation times of the papers published from Chinese institutions also ranked first. These accomplishments were attributable to the contribution of young scientists in China.

In June 2017, 36 young scientists were invited to join Chinese Journal of Catalysis as the Young Members of the Editorial Board. Undoubtedly, their participation will further enhance the quality and impact of Chinese Journal of Catalysis. To celebrate the growing and thriving of Chinese Journal of Catalysis through the admission of these the Young Members of the Editorial Boards, we publish this special issue Photocatalysis in China because many members' work is related to photocatalysis.

Twenty-three papers are collected in this special issue. Among them, two papers are review papers. Most of papers were contributed by these the Young Members of the Editorial Boards. All the published papers have been reviewed according to the normal review practices of Chinese Journal of Catalysis. As the Guest Editor of this special issue, I would like to express my sincere thanks to all the authors who contributed to this special issue and all the Reviewers for timely evaluation of the submitted manuscripts. Especially, I appreciate the warm invitation and kind assistance from the Editorial Office Staff of Chinese Journal of Catalysis for the publication of this special issue.

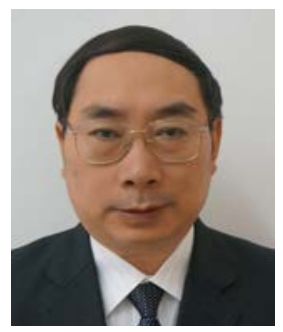

Prof. Jiaguo Yu (Guest Editor)

State Key Laboratory of Advanced Technology for Material Synthesis and Processing, Wuhan University of Technology, Wuhan, China E-mail address: jiaguoyu@yahoo.com 